\title{
Extended Gradient Predictor and Filter for Smoothing RSSI
}

\author{
Fazli Subhan ${ }^{1}$, Salman Ahmed $^{2}$ and Khalid Ashraf ${ }^{3}$ \\ ${ }^{1}$ Department of Information Technology and Engineering, \\ National University of Modern Languages-NUML, Islamabad, Pakistan \\ ${ }^{2}$ Department of Electrical Engineering, Bahria University Islamabad, Pakistan \\ ${ }^{3}$ Department of Electrical and Electronic Engineering, \\ Universiti Teknologi PETRONAS, Malaysia \\ fazli.subhan@numl.edu.pk, salmanelectric@gmail.com, ashraf.balghari@gmail.com
}

\begin{abstract}
This paper presents the design of an Extended Gradient Predictor and Filter for smoothing Received Signal Strength Indicator (RSSI). In wireless communication RSSI is a measurement of power in received signal, which is a parameter used for handover and position estimation purpose. In real time applications such as handover and position estimation requires continuous reception of RSSI measurements. Due to the effect of noise, variations occur in the received signal, which sometimes produce communication holes. In that case, RSSI measurements become zero, which produces distance estimation error. In order to minimize the effect communication, an existing Gradient filter is modified based on most recent RSSI measurement received. The advantage of the proposed extended Gradient RSSI predictor and filter is that it predicts and filters RSSI values in the presence of communication holes for real time object tracking. The performance of proposed extended Gradient RSSI predictor and filter is tested using simulated and real-time RSSI measurements using Bluetooth technology, which shows better performance in case of communication holes occur.
\end{abstract}

Index Terms-: Gradient Filter, RSSI, Communication holes, Position estimation

\section{INTRODUCTION}

Due to the rapid advancements in wireless communication and mobile technologies, Location based services is considered to be an important aspect of mobile technology. In today's modern and technological world, position estimation plays an important role in all aspects of people's daily lives.In position estimation, accuracy is one of the key performance indicators for indoor position estimation. In real-time object tracking applications, continuous reception of RSSI measurements is mandatory so that exact position of the target node at each time instant can be identified [1], [2]. In Radio Frequency (RF) based wireless communication, position estimation is performed using RSSI, which is the parameter used to estimate distance between mobile and anchor nodes or APs [3], [4]. Currently most of the existing wireless technologies use RF waves for communication such as Wireless Local Area Network (WLAN), Bluetooth and Zigbee [5], [6].

The use of RF sensing technology for indoor position estimation techniques introduces some challenges such as the effect of signal attenuation and multi-path effects, which occur due to the reflections of signals in indoor environments [7].
In this paper Bluetooth technology is considered for position estimation, therefore, the issues related to position estimation will be discussed. In Bluetooth networks, the inquiry procedure takes around 10.24 seconds to discover the nearby devices [8], [9]. In real-time applications involving position estimation, the time taken to identify the position of a target node is also an important design objective, which relies on continuous reception of RSSI measurements [10]. In case of Bluetooth technology, the inquiry-based RSSI suffers from GRPR zero value problem [11], [12]. Moreover, various environmental conditions can effect the transmission and reception of RSSI such as increase of temperature, reflection of sound waves and presence of human bodies [13]. All these factors cause the transmitted signal to attenuate and become very weak. Hence, it appears to the receiver that a disconnection has occurred. This disconnection between transmitter and receiver produces communication holes, which negatively effects the accuracy of estimated position .

In order to improve the accuracy of estimated position, a filter is needed which is used to filter the RSSI measurements [14]. The objective of the filter is to minimize the effects of communication holes. The proposed extended Gradient RSSI predictor and filter is used to predict RSSI measurements when frequent disconnections occur. The advantage of proposed extended approach is that it predicts RSSI values of a moving object based on the current movement of the object. If the object is moving towards the master node, then probably the numeric values of RSSI measurements would increase, and if the object is moving away from the master node, then the RSSI values would decrease proportionally. Therefore, the increase and decrease in RSSI values indicate the current movement of user [15]. Hence, the most recent RSSI measurements received demonstrate the current movement.

The remainder of this paper is structured as follow. Section II discusses the design of our proposed extended Gradient RSS predictor and Filter, Section III presents a Comparative analysis of the Proposed extended Gradient RSS predictor and filter, Section IV, presents applications of the proposed Gradient filter and Finally conclusion and future research directions are presented in Section V. 


\section{Design of Proposed Extended GRAdient RSSI PREDICTOR AND FILTER}

Consider a mobile client with Bluetooth enabled device, which is moving inside the communication range of Bluetooth APs, where each location is associated with a specific RSSI value. Let $x$ represents the RSSI values observed at time $t_{i}$, which can be expressed as follows:

$$
x=\left[x_{1}, x_{2}, x_{3}, \ldots, x_{n}\right],
$$

where $x_{i}$ for $\mathrm{i}=1, \ldots, n$ represents the RSSI measurements at time instant $t_{i}$. The time vector containing the time-stamps $t_{i}$, for $\mathrm{i}=1, \ldots, n$ can be written as follows:

$$
t=\left[t_{1}, t_{2}, t_{3}, \ldots, t_{n}\right] .
$$

Let the time between two successful measurements be denoted as $\Delta t_{j}$, which can be expressed as follows:

$$
\Delta t_{j}=t_{j}-t_{j-1}, j=1, \ldots, n
$$

It should be noted that the values of $\Delta t_{j}$ are not the same for each time instant as the measurements may arrive or may not arrive due to communication holes. Let $\Delta t_{n}$ represent the time interval in which the most recent successful reception of the signal occurred. If the mobile client is having a non-uniform speed, then the rate of change of RSSI measurements will also be varying. At any time instant $t_{i}$, the instantaneous rate of change of RSSI values can be written as follows:

$$
R_{i}=\frac{\Delta x_{i}}{\Delta t_{i}}=\frac{x_{i}-x_{i-1}}{t_{i}-t_{i-1}}
$$

where $R_{i}$ denotes the instantaneous rate of change of RSSI values with respect to time. The original Gradient predictor and filter considers all of the previous values till time $t_{n}$, and maintains a window size of $n$ measurements. In the proposed extended Gradient RSSI predictor and filter, the window size keeps $k$ measurements. The size of $k$ depends on the number of measurements used to calculate the previous rate of change. Consider the RSSI value $x_{n}$ measured at time instant $t_{n}$, and a window size of $k$, then the most recent average rate of change at the time instant $t_{n}$ can be written as follows:

$$
R_{n}=\frac{1}{k} \sum_{i=n-k}^{n-1} R_{i},
$$

where $R_{n}$ represents the most recent average rate of change. Having this representation, the $n^{\text {th }}$ predicted RSSI value can be expressed as follows:

$$
X_{n}=\left(\frac{1}{k} \sum_{i=n-k}^{n-1} R_{i} * \Delta t_{n}\right)+x_{n-1},
$$

where $X_{n}$ represents the predicted RSSI value. The prediction process is used to predict the next RSSI measurement. In order to minimize the variations in RSSI due to various environmental conditions, the measurements are filtered by applying standard deviation rule. The following equation is used to calculate the standard deviation of the predicted RSSI and previous RSSI value.

$$
\sigma\left(X_{n}, x_{n}\right)=\sqrt{\frac{1}{2}\left(X_{n}-x_{n}\right)^{2}}
$$

where $\sigma\left(X_{n}, x_{n}\right)$ represents the standard deviation between the predicted $X_{n}$ and the current $x_{n}$. By using Eq. 7, the filtered RSSI measurements can be obtained by the following representation [16].

$$
x_{f}=X_{n}+\left(\operatorname{sign}\left(X_{n}-x_{n}\right)\right) * \sigma\left(X_{n}, x_{n}\right) * \sigma\left(X_{n}, x_{n}\right)^{2},
$$

where sign() extracts positive or negative sign of the difference between predicted $X_{n}$ and the current received RSSI measurement $x_{n}$. The output of $\operatorname{sign()~will~be~} 1$ if the input is positive and -1 if the input is negative and zero if the input is zero. The modifications in the proposed extended Gradient RSSI predictor and filter is performed by changing the window size. The original Gradient filter maintains a window size of $n$ RSSI measurements and the proposed design only maintains a window size of $k$ RSSI measurements. In order to analyze the performance of proposed extended Gradient RSSI predictor and filter, it is compared with the original Gradient filter and Kalman filter. The following sub-section presets an analysis of the proposed filter using simulated and real-time RSSI measurements.

\section{Comparative Analysis of the Proposed} EXTENDED GRADIENT RSSI PREDICTOR AND FILTER VS. ORIGINAL GRADIENT FILTER

The effectiveness of the proposed extended Gradient RSSI predictor and filter is presented by comparing it with the original Gradient filter using ideal and simulated RSSI measurements. Ideal RSSI measurements reflect the real-time RSSI measurements obtained with respect to time, while simulated RSSI measurements reflect the presence of communication holes. The phenomenon of communication holes is represented by zero RSSI measurements, which indicates the delay in received signal or missing received signal at the receiver side. In order to check performance of the proposed extended Gradient RSSI predictor and filter, the delay occurs in the transmission of RSSI measurements or interruption occurs due to noise, which indicates communication holes. Therefore, the numerical value for representing the communication holes is referred as zero. The reason behind simulated RSSI measurements is to see the performance of proposed extended Gradient filter in presence of communication holes. The performance of proposed extended Gradient filter is also compared with the original Gradient filter. The numerical results are presented based on four different possible scenarios. In all fours scenarios, it is assumed that the receiver collects one RSSI sample in one second, which corresponds to the movement of user. Normally a human body moves at a speed of 1 meter/sec [16]. The proposed extended Gradient RSSI predictor and filter can be equally applied to irregular movement of the body in which speed of the user is not uniform. The performance of the proposed extended Gradient is compared with the ideal RSSI measurements collected for each scenario, which is the average value of 10 RSSI measurements. 
In Bluetooth technology, the inquiry procedure takes around 10.24 seconds to discover nearby Bluetooth devices. The program is developed to inquire RSSI measurements using BlueZ, which is a Bluetooth programming language developed to communicate with Bluetooth devices. The Application programming Interface (API) used to inquire RSSI is BlueZ D-BUS () in which the inquiry delay to discover Bluetooth devices is decreased in such a way that the RSSI measurements are inquired every second. The idea behind this approach is to extract RSSI at every second corresponding to the movement of human body.

Fig. 1 represents case 1 in which the mobile node is moving away from master node. The ideal RSSI measurements show the average RSSI measurements collected, when the mobile node is moving at a speed of $1 \mathrm{~m} / \mathrm{sec}$. To see performance of the proposed extended Gradient RSSI predictor and filter, it is compared with original Gradient filter. The simulated RSSI measurements represent the case if there is a communication hole. Compared to the ideal RSSI values, the performance of proposed extended Gradient RSSI predictor and filter is better than original Gradient filter. In case 1, a total of 4 communication holes are inserted, one in the middle and three consecutive communication holes before the last measurement received. The value zero represents presence of communication hole. The ideal RSSI measurements were collected when mobile node is going away from the master node from 1 to 10 meters. Three consecutive communications holes were inserted at a distance of 7, 8 and 9 meters, which represented missing RSSI measurements. The prediction process is performed based on the most recent RSSI measurements received.

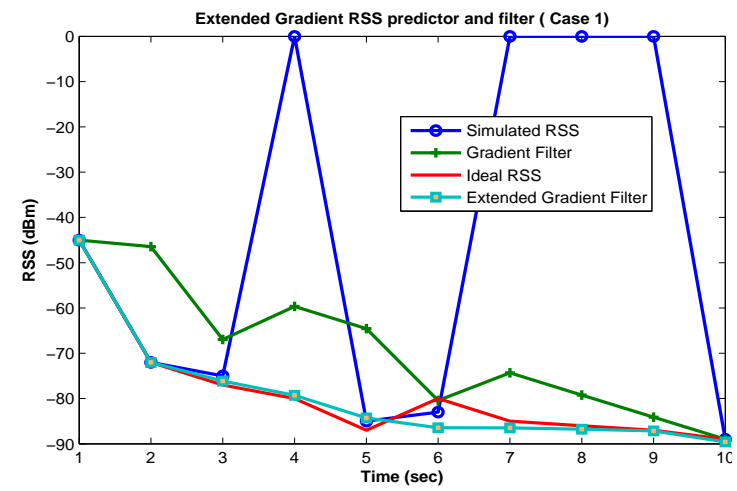

Fig. 1. Plot of RSSI measurements which are filtered using the proposed and actual Gradient filter (Case 1).

According to the experimental observations the maximum reported RSSI value is zero, which means the presence of communication hole while the minimum RSSI value is -90 $\mathrm{dBm}$. The prediction process is performed based on the value of $k=1$. If the value of $k$ is 2 , then the prediction process is performed based on previous 2 values and the $3^{\text {rd }}$ value is predicted. The value of $k$ can be changed accordingly. When the value is predicted, so it is then filtered by measuring standard deviation of predicted and most recently received RSSI values.
Fig. 2 represents case 2 , when the mobile node is at a distance of 7 meters from master node moving along the circumference with a radius of 7 meters. In this scenario the mobile node is moving at distance of 7 meters along the circumference of a circle with a radius of 7 meters. The movement of mobile node is not uniform which indicates the mobile user can stay at one position or move fast or slow. The ideal RSSI measurements are collected accordingly with the distance of 7 meters with respect to time and average values are calculated for comparison. The communication holes are inserted at different positions. Compared to the ideal RSSI measurements, performance of the proposed extended Gradient RSSI predictor and filter is better than original Gradient filter. In Fig. 2 the last RSSI value is zero, which means there is a communication hole, so the proposed extended Gradient filter predicts the last value based on the previous two values. However, the original Gradient filter predicts RSSI value near to zero because it considers the $n$ RSSI values. Therefore, performance of the extended Gradient filter is much better than original Gradient filter.

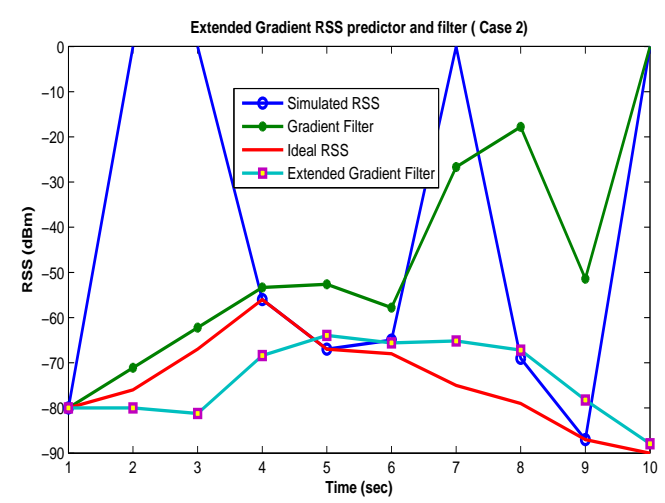

Fig. 2. Plot of RSSI measurements which are filtered using the proposed and actual Gradient filter (Case 2).

Fig. 3 represents case 3 , in which the mobile client stays at a distance of 6 meters away from the master device. The reason behind collecting the RSSI measurements at a distance of 6 meters is to identify the variations in RSSI with respect to time when mobile node is at fixed position. Communication holes appear at any time due to noise or delay in the inquiry process. A physical object is placed in front of the mobile node to observe fluctuations in RSSI due to line-of-sight problem. The ideal RSSI measurements were obtained and it is observed that the inquiry procedure fetched RSSI measurements with more than 1 second. Due to this delay, some of the Bluetooth devices are not discovered in inquiry procedure and hence the returned RSSI value is zero or considered as zero. In case 3, the ideal RSSI measurements decrease from 1 to 4 seconds, therefore the prediction process is performed based on the real-time rate of change occurrence. Fig. 3 indicates that the proposed extended Gradient filter performs better than original Gradient filter. The proposed extended Gradient filter predicts the new RSSI measurements which is almost similar to the ideal RSSI measurements. 


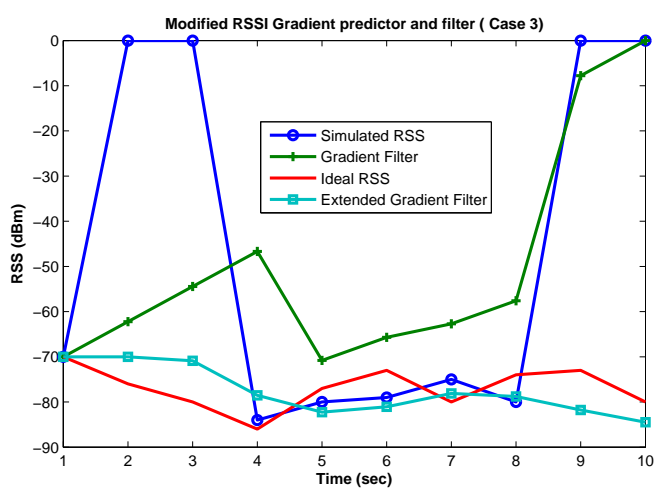

Fig. 3. Plot of RSSI measurements which are filtered using the proposed and actual Gradient filter (Case 3).

The above case considers the movement of mobile client in presence of communication holes. In all of above three cases, the movement of mobile client is not fixed or constant. In previous research [16], the movement of mobile client is constant while in this case movement of mobile client is unknown. Here, prediction is performed based on the most recent RSSI value observed, which represents the current speed of mobile client. The main contribution of this approach is prediction process which is based on the current movement of mobile client. If the mobile client is moving towards the master node so the values of RSSI will be increasing, in case moving away so the values of RSSI will be decreasing. The communication holes can appear at any instant of time, which do not depend on the speed of mobile client. Therefore, compared to previous research on Gradient filter the proposed extended Gradient filter is better and applicable for real-time scenarios.

Fig. 4 represents case 4 , when no communication holes are observed. The distance between mobile and master node is varied from 5 meters to 1 meters. The ideal RSSI measurements were calculated when the mobile node is coming towards the master node. The mobile and master node are in lineof-sight and can communicate without interruption. In this case, the performance of proposed extended Gradient filter depends on the value of RSSI and its standard deviation. The filtering process is based on the most recent predicted value. In ideal conditions, if mobile and master node are in the lineof-sight then signal attenuation is minimum. Fig. 4 shows the performance of Gradient filter in case of no communication holes. The mobile node is within the communication range of master node and there is no object in the middle. The conditions are considered as an ideal state for the Gradient filter. The ideal and simulated RSSI measurements are given to the original and proposed extended Gradient filter to see performance of the techniques. In Fig. 4 it is clear that the proposed extended Gradient filter predicts the RSSI values comparatively better than the original Gradient filter. However, the variations in predicted RSSI measurements are lesser than ideal measurements.

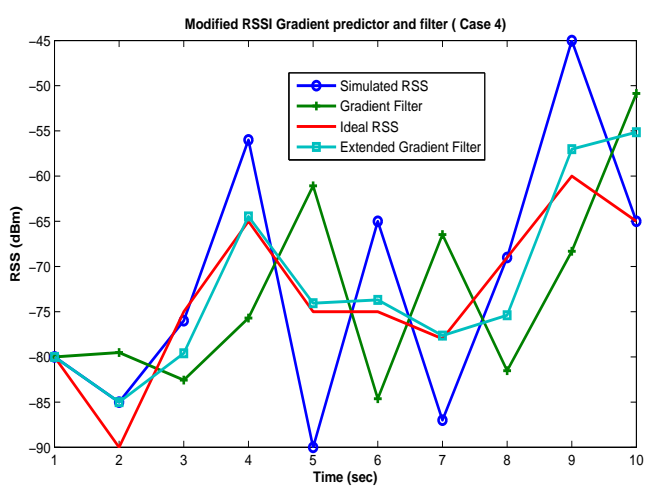

Fig. 4. Plot of RSSI measurements which are filtered using the proposed and actual Gradient filter (Case 4).

The main idea behind modeling the Gradient RSSI predictor and filter is to minimize the issue of communication holes. If there is no communication hole then Kalman filter can be used, which is a standard mathematical model designed to predict RSSII measurements in the presence of noisy environment. However, due to limitations of Kalman filter in case of communication holes, there is a dire need to address the issue of communication holes [17]. The basic assumption to use Kalman filter is to know the statistical properties of noise. Kalman filter performs better if the properties of noise are known. The second most common assumption of Kalman filter is that it minimizes the mean error only. However, in case of worst kind of error such as communication hole, Kalman filter will not be able to work properly [17].

In order to compare performance of the proposed extended Gradient filter, Kalman filter is implemented and the same set of RSSI measurements are processed using Kalman filter and proposed extended Gradient filter. For comparisons, the average RSSI measurements are observed, which are considered as an ideal RSSI measurements.

\section{Applications of Proposed Extended Gradient RSSI PREDICTOR AND FILTER}

The scope of proposed extended Gradient filter is not limited to the position estimation techniques, where accurate and timely measurement of the received signal is crucial. For example seamless handover from one Access Point (AP) to another AP is an application of the proposed extended Gradient RSSI predictor and filter. The term handover refers to situation in which a mobile node transfers an ongoing connection from one to another AP without disconnection with the previous AP. In case of handover prediction, when the mobile node is moving out of coverage zones or the area where effect of noise is more, then the received signals become weak. Therefore, the prediction process initiates handover if the received signal becomes lesser than threshold value, which is defined for handover activation. The initiation of handover process depends on the value of received signal. In case of communication hole, which occurs due to many factors such as weak communication 
signal, noise or handover zones, the communication between master and mobile node will be disconnected. So in case of frequent disconnections, the proposed extended Gradient filter can be used to predict and filter the received signals in order to maintain communication between master and mobile node.

\section{CONClusions And Future RESEARCH DiRECTION}

Accuracy is one of the most important aspect in position estimation both for indoor and outdoor applications. For indoor positioning system, accurate and timely measurement of RSSI is required. The main objective of modeling the signal behavior is to improve the position estimation accuracy. This paper presented an extended Gradient RSSI predictor and filter. The performance of the proposed extended Gradient filter is analyzed through real-time and simulated RSSI measurements and compared with Kalman filter and Kalman smoother. The numerical results presented in this paper conclude that the performance of proposed extended Gradient filter is better than Kalman filter and Kalman smoother in case of communication holes. The major contribution of this paper is the design of an extended Gradient RSSI predictor and filter. The objective of modeling the Gradient filter is to predict and filter the RSSI values in presence of communication holes and other environmental conditions which produce variations in RSSI. Future research is needed to propose other mathematical models to handle the issue of communication holes.

\section{REFERENCES}

[1] J. Hightower and G. Borriella, "Location systems for ubiquitous computing," IEEE Computer, vol. 34, no. 8, pp. 57-66, 2001.

[2] D. Zhang, F. Xia, Z. Yang, L. Yao, and W. Zhao, "Localization Technologies for Indoor Human Tracking," in $5^{\text {th }}$ International Conference Future Information Technology (FutureTech), pp. 1-6, 2010.

[3] L. Dong and W. Jiancheng, "Research of indoor local positioning based on bluetooth technology," in Proceedings of the 5th International Conference on Wireless communications, networking and mobile computing, pp. 5211-5214, 2009.

[4] R. Rong and M. L. Sichitiu, "Indoor localization system using rssi measurement of wireless sensor network based on zigbee standard," in IASTED International Conference on Wireless Sensor Networks, Alberta, Canada, pp. 1-6, 2006.

[5] H. Liu, H. Darabi, P. Banerjee, and J. Liu, "Survey of wireless indoor positioning techniques and systems," IEEE Transactions on Systems, Man, and Cybernetics, Part C, vol. 37, pp. 1067-1080, 2007.

[6] S. Pandey and P. Agrawal, "A survey on localization techniques for wireless networks," Journal of the Chinese Institute of Engineers, vol. 29, no. 7, pp. 1125-1148, 2007.

[7] F. Subhan, H. Hasbullah, A. Rozyyev, and S. T. Bakhsh, "Indoor positioning in bluetooth networks using fingerprinting and lateration approach," in Information Science and Applications (ICISA), 2011 International Conference on, pp. 1-9, IEEE, 2011.

[8] Bluetooth Special Intrest Group, "Fast-facts @ONLINE," 2011.

[9] A. Pramod-Patil, "Performance of bluetooth technologies and their applications to location sensing," Master's thesis, Department of Electrical and Computer Engineering, Michigan State University, USA, 2002.

[10] F. Subhan, H. Hasbullah, and K. Ashraf, "Kalman filter-based hybrid indoor position estimation technique in bluetooth networks," International Journal of Navigation and Observation, vol. 2013, 2013.

[11] M. Hossain and S. Wee-Seng, "A comprehensive study of bluetooth signal parameters for localization," in IEEE International Symposium on Personal, Indoor and Mobile Radio Communications, pp. 187-192, 2007.

[12] C. D. Dimitrova, U. Burgi, G. Martins, and T. Braun, "Inquiry-based bluetooth parameters for indoor localisation-an experimental study," in 5th ERCIM Workshop on eMobility,European Research Consortium for Informatics and Mathematics, pp. 1-9, 2011.
[13] X. Luo, W. Brien, and C. Julien, "Comparative evaluation of received signal-strength indicator (rssi)-based indoor localization techniques for construction jobsites," Advanced Engineering Informatics, vol. 25, no. 2, pp. $355-363,2011$.

[14] F. Subhan, H. Hasbullah, A. Rozyyev, and S. T. Bakhsh, "Analysis of bluetooth signal parameters for indoor positioning systems," in Computer \& Information Science (ICCIS), 2012 International Conference on, vol. 2, pp. 784-789, IEEE, 2012.

[15] F. Subhan, H. Hasbullah, A. Rozyyev, and S. T. Bakhsh, "Handover in bluetooth networks using signal parameters," Information Technology Journal, vol. 10, no. 5, pp. 965-973, 2011.

[16] K. A. Elbatsh, L. E. Macas, and A. S. Sarmiento, "Rssi gradient new predictor and filter to support sporadic wireless service interruptions," in $7^{\text {th }}$ IASTED International Conference Communication Systems and Networks, Palma de Mallorca, Spain, pp. 1-9, 2008.

[17] K. A. Elbatsh, L. E. Macas, and A. S. Samiento, "Gradient rssi filter and predictor for wireless network algorithms and protocols," Network Protocols and Algorithms, vol. 2, no. 2, pp. 1-26, 2010. 